\title{
Rainbow Hamilton cycles in uniform hypergraphs
}

\author{
Andrzej Dudek \\ Department of Mathematics \\ Western Michigan University \\ Kalamazoo, MI \\ andrzej .dudek@wmich.edu
}

\author{
Alan Frieze* \\ Department of Mathematical Sciences \\ Carnegie Mellon University \\ Pittsburgh, PA \\ alan@random.math.cmu.edu
}

\author{
Andrzej Ruciński ${ }^{\dagger}$ \\ Department of Discrete Mathematics \\ Adam Mickiewicz University \\ Poznań, Poland \\ rucinski@amu.edu.pl
}

Submitted: Sep 7, 2011; Accepted: Feb 8, 2012; Published: Feb 23, 2012

Mathematics Subject Classifications: 05C15, 05C65

\begin{abstract}
Let $K_{n}^{(k)}$ be the complete $k$-uniform hypergraph, $k \geq 3$, and let $\ell$ be an integer such that $1 \leq \ell \leq k-1$ and $k-\ell$ divides $n$. An $\ell$-overlapping Hamilton cycle in $K_{n}^{(k)}$ is a spanning subhypergraph $C$ of $K_{n}^{(k)}$ with $n /(k-\ell)$ edges and such that for some cyclic ordering of the vertices each edge of $C$ consists of $k$ consecutive vertices and every pair of adjacent edges in $C$ intersects in precisely $\ell$ vertices.

We show that, for some constant $c=c(k, \ell)$ and sufficiently large $n$, for every coloring (partition) of the edges of $K_{n}^{(k)}$ which uses arbitrarily many colors but no color appears more than $c n^{k-\ell}$ times, there exists a rainbow $\ell$-overlapping Hamilton cycle $C$, that is every edge of $C$ receives a different color. We also prove that, for some constant $c^{\prime}=c^{\prime}(k, \ell)$ and sufficiently large $n$, for every coloring of the edges of $K_{n}^{(k)}$ in which the maximum degree of the subhypergraph induced by any single color is bounded by $c^{\prime} n^{k-\ell}$, there exists a properly colored $\ell$-overlapping Hamilton cycle $C$, that is every two adjacent edges receive different colors. For $\ell=1$, both results are (trivially) best possible up to the constants. It is an open question if our results are also optimal for $2 \leq \ell \leq k-1$.

The proofs rely on a version of the Lovász Local Lemma and incorporate some ideas from Albert, Frieze, and Reed.
\end{abstract}

*Supported in part by NSF grant CCF1013110.

${ }^{\dagger}$ Supported in part by the Polish NSC grant N201 604940 and the NSF grant DMS-1102086. 


\section{Introduction}

By a coloring of a hypergraph $H$ we mean any function $\phi: H \rightarrow \mathbb{N}$ assigning natural numbers (colors) to the edges of $H$. (In this paper we do not consider vertex colorings.) A hypergraph $H$ together with a given coloring $\phi$ will be dubbed a colored hypergraph. A subhypergraph $F$ of a colored hypergraph $H$ is said to be properly colored if every two adjacent edges of $F$ receive different colors. (Two different edges are adjacent if they share at least one vertex.) We say that a subhypergraph $F$ of a colored hypergraph $H$ is rainbow if every edge of $F$ receives a different color, that is, when $\phi$ is injective on $F$.

In order to force the presence of properly colored or rainbow subhypergraphs one has to restrict the colorings $\phi$, either globally or locally. A coloring $\phi$ is $r$-bounded if every color is used at most $r$ times, that is, $\left|\phi^{-1}(i)\right| \leq r$ for all $i \in \mathbb{N}$. A coloring $\phi$ is $r$-degree bounded if the hypergraph induced by any single color has maximum degree bounded by $r$, that is, $\Delta\left(H\left[\phi^{-1}(i)\right]\right) \leq r$ for all $i \in \mathbb{N}$.

In this paper we study the existence of properly colored and rainbow Hamilton cycles in colored $k$-uniform complete hypergraphs, $k \geq 3$. (A hypergraph is $k$-uniform if every edge has size $k$; it is complete if all $k$-element subsets of the vertices form edges.) There is a broad literature on this subject for $k=2$, that is, for graphs. Indeed, setting $r=c n$, Alon and Gutin proved in [2], improving upon earlier results from [5, 6, 16] that if $c<1-1 / \sqrt{2}$ then any $r$-degree bounded coloring of the edges of the complete graph $K_{n}$ yields a properly colored Hamilton cycle (for the history of the problem, see [3]). It had been conjectured in [5] that the constant $1-1 / \sqrt{2}$ can be replaced by $\frac{1}{2}$ which is the best possible.

Rainbow Hamilton cycles in $r$-bounded colorings of the complete graph have been studied in $[1,8,10,12]$. Hahn and Thomassen conjectured that their existence is guaranteed if $r=c n$ for some $c>0$. This was confirmed by Albert, Frieze, and Reed in [1] with $c=\frac{1}{64}$. Again, $c=1 / 2$ seems to be a critical value here, since one can use each of $n-1$ colors exactly $n / 2$ times, making the presence of rainbow Hamilton cycles impossible. In striking contrast, there is literally nothing known on properly colored or rainbow Hamilton cycles in colored $k$-uniform hypergraphs for $k \geq 3$.

The notion of a hypergraph cycle can be ambiguous. In this paper we are not concerned with the Berge cycles as defined by Berge in [4] (see also [11]). Instead, following a recent trend in the literature $([7,13,15])$, given an integer $1 \leq \ell<k$, we define an $\ell$-overlapping cycle as a $k$-uniform hypergraph in which, for some cyclic ordering of its vertices, every edge consists of $k$ consecutive vertices, and every two consecutive edges (in the natural ordering of the edges induced by the ordering of the vertices) share exactly $\ell$ vertices. (See Fig. 1 for an example of a 2-overlapping and a 3-overlapping 5-uniform cycle.)

The two extreme cases of $\ell=1$ and $\ell=k-1$ are referred to as, respectively, loose and tight cycles. Note that the number of edges of an $\ell$-overlapping cycle with $s$ vertices is $s /(k-\ell)$. Note also that when $k-\ell$ divides $s$, every tight cycle on $s$ vertices contains an $\ell$-overlapping cycle on the same vertex set (with the same cyclic ordering).

Given a $k$-uniform hypergraph $H$ on $n$ vertices, where $k-\ell$ divides $n$, an $\ell$-overlapping cycle contained in $H$ is called Hamilton if it goes through every vertex of $H$, that is, if 

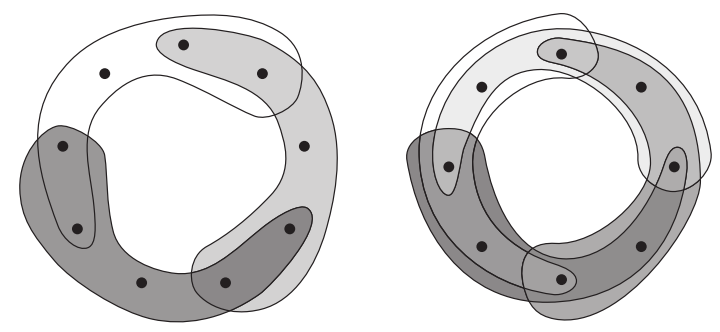

Figure 1: A 2-overlapping and a 3-overlapping 5-uniform cycle.

$s=n$. We denote such a Hamilton cycle by $C_{n}^{(k)}(\ell)$.

In this paper we prove the following two results. Let $K_{n}^{(k)}$ be the complete $k$-uniform hypergraph of order $n$.

Theorem 1.1 For every $1 \leq \ell<k$ there is a constant $c=c(k, \ell)$ such that if $n$ is sufficiently large and $k-\ell$ divides $n$ then any $c n^{k-\ell}$-bounded coloring of $K_{n}^{(k)}$ yields a rainbow copy of $C_{n}^{(k)}(\ell)$.

Theorem 1.2 For every $1 \leq \ell<k$ there is a constant $c^{\prime}=c^{\prime}(k, \ell)$ such that if $n$ is sufficiently large and $k-\ell$ divides $n$ then any $c^{\prime} n^{k-\ell}$-degree bounded coloring of $K_{n}^{(k)}$ yields a properly colored copy of $C_{n}^{(k)}(\ell)$.

Note that for loose Hamilton cycles (i.e. $\ell=1$ ) the above results are optimal up to the values of $c$ and $c^{\prime}$. Theorem 1.2 is trivially optimal, as the largest maximum degree can be at most $r=\left(\begin{array}{l}n-1 \\ k-1\end{array}\right) \sim \frac{1}{(k-1) !} n^{k-1}$. To see that also Theorem 1.1 is optimal up to the constant for $\ell=1$ consider any coloring of $K_{n}^{(k)}$ using each color precisely

$$
r=\frac{\left(\begin{array}{l}
n \\
k
\end{array}\right)}{\frac{n}{k-1}-1} \sim \frac{k-1}{k !} n^{k-1}
$$

times, and thus using only $\frac{n}{k-1}-1$ colors altogether. Such a coloring is $r$-bounded and, clearly, there is no rainbow copy of $C_{n}^{(k)}(1)$.

Problem 1.3 For all $k \geq 3$ and $\ell=1$, determine $\sup c$ and $\sup c^{\prime}$ over all values of $c$ and, respectively, $c^{\prime}$ for which Theorems 1.1 and 1.2 hold.

We believe that Theorems 1.1 and 1.2 are optimal up to the constants also for $\ell \geq 2$, that is, we believe that the answer to the following question is positive.

Problem 1.4 For all $k \geq 3$ and $2 \leq \ell \leq k-1$, does there exist an $r$-bounded $(r$-degree bounded) coloring $\phi$ of $K_{n}^{(k)}$ such that $r=\Theta\left(n^{k-l}\right)$ and no copy of $C_{n}^{(k)}(\ell)$ is rainbow (properly colored)?

As some evidence supporting our belief, consider the bipartite version of both problems for $k=3$ and $\ell=2$. Let $K_{n, 2 n}^{(3)}=\left(V_{1}, V_{2}, E\right)$, where $\left|V_{1}\right|=n,\left|V_{2}\right|=2 n$ and $E=\{e \subset$ $\left.V_{1} \cup V_{2}:\left|e \cap V_{i}\right|=i, \quad i=1,2\right\}$. To every edge $e$ assign the pair $e \cap V_{2}$ as its color. Clearly, every color appears exactly $n$ times and hence such a coloring is $n$-bounded (and 
thus $n$-degree bounded). Finally, note that every tight Hamilton cycle in $K_{n, 2 n}^{(3)}$ induces a cyclic sequence of vertices with a repeated pattern of two vertices from $V_{2}$ followed by one vertex from $V_{1}$. Hence, there is a pair of consecutive edges with the same color (actually, there are $n$ such pairs), and so no copy of a properly colored (or rainbow) $C_{n}^{(3)}(2)$ exists.

\section{The proofs}

We will need a special version of the Lovász Local Lemma. A similar result was already established in $[1,9,14]$. Contrary to the above results, in our formulation of the lemma we avoid conditional probabilities so that we do not need to make a priori assumptions that certain events have positive probability.

Lemma 2.1 Let $A_{1}, A_{2}, \ldots, A_{m}$ be events in an arbitrary probability space $\Omega$. For each $1 \leq i \leq m$, let $[m] \backslash\{i\}=X_{i} \cup Y_{i}$ be a partition of the index set $[m] \backslash\{i\}$ and let

$$
d=\max \left\{\left|Y_{i}\right|: 1 \leq i \leq m\right\} .
$$

If for each $1 \leq i \leq m$ and all $X \subseteq X_{i}$

$$
\operatorname{Pr}\left(A_{i} \cap \bigcap_{j \in X} \overline{A_{j}}\right) \leq \frac{1}{4(d+1)} \operatorname{Pr}\left(\bigcap_{j \in X} \overline{A_{j}}\right)
$$

then $\operatorname{Pr}\left(\bigcap_{i=1}^{m} \overline{A_{i}}\right)>0$. (We adopt the convention that $\bigcap_{j \in \emptyset} \overline{A_{j}}=\Omega$.)

Proof. We prove by induction on $t=1, \ldots, m$, that for every $T \subseteq[m],|T|=t$, and for every $i \in T$, setting $S=T \backslash\{i\}$, we have

$$
\operatorname{Pr}\left(\bigcap_{i \in T} \overline{A_{i}}\right)>0 \quad \text { and } \operatorname{Pr}\left(A_{i} \mid \bigcap_{i \in S} \overline{A_{i}}\right) \leq \frac{1}{2(d+1)} .
$$

For $t=1$ we apply (2) with $X=\emptyset$, obtaining for each $i$ that $\operatorname{Pr}\left(A_{i}\right) \leq \frac{1}{4(d+1)}$, equivalently $\operatorname{Pr}\left(\overline{A_{i}}\right) \geq 1-\frac{1}{4(d+1)}>0$, which confirms $(3)$ for $t=1$.

Now, assume truth for some $t, 1 \leq t \leq m-1$, and consider a set $T=\{i\} \cup S$, where $i \notin S$ and $|S|=t$. Set $X=S \cap X_{i}$ and $Y=S \cap Y_{i}$, and observe that $S=X \cup Y$ and $|Y| \leq\left|Y_{i}\right| \leq d$. By the induction assumption $\operatorname{Pr}\left(\bigcap_{j \in S} \overline{A_{j}}\right)>0$. If $Y=\emptyset$ (and thus $X=S)$, by our assumption (2),

$$
\operatorname{Pr}\left(A_{i} \mid \bigcap_{j \in S} \overline{A_{j}}\right) \leq \frac{1}{4(d+1)}
$$

Otherwise, $|X|<|S|=t$ and, again by (2) (in the numerator) and the induction assumption (in the denominator) we argue that

$$
\operatorname{Pr}\left(A_{i} \mid \bigcap_{j \in S} \overline{A_{j}}\right)=\frac{\operatorname{Pr}\left(A_{i} \cap \bigcap_{j \in Y} \overline{A_{j}} \mid \bigcap_{j \in X} \overline{A_{j}}\right)}{\operatorname{Pr}\left(\bigcap_{j \in Y} \overline{A_{j}} \mid \bigcap_{j \in X} \overline{A_{j}}\right)} \leq \frac{\frac{1}{4(d+1)}}{1-|Y| \frac{1}{2(d+1)}} \leq \frac{1}{2(d+1)} .
$$


Thus,

$$
\operatorname{Pr}\left(\bigcap_{j \in T} \overline{A_{j}}\right)=\operatorname{Pr}\left(\overline{A_{i}} \mid \bigcap_{j \in S} \overline{A_{j}}\right) \operatorname{Pr}\left(\bigcap_{j \in S} \overline{A_{j}}\right)>0,
$$

which completes the proof of Lemma 2.1 .

The proofs of Theorems 1.1 and 1.2 extend some ideas introduced by Albert, Frieze and Reed in [1].

Proof of Theorem 1.1. Fix $1 \leq \ell<k$ and a $c n^{k-\ell}$-bounded coloring $\phi$ of $K_{n}^{(k)}$ for some constant $c>0$ to be specified later. Define

$$
M=\left\{\{e, f\}: e, f \in K_{n}^{(k)}, \quad|e \cap f| \leq \ell \text { and } \phi(e)=\phi(f)\right\} .
$$

Moreover, for every pair $\{e, f\} \in M$ set

$$
A_{e, f}=\left\{C \subset K_{n}^{(k)}: C \cong C_{n}^{(k)}(k-1) \text { and }\{e, f\} \subset C\right\} .
$$

In order to prove Theorem 1.1 it suffices to show that

$$
\bigcap_{\{e, f\} \in M} \overline{A_{e, f}} \neq \emptyset .
$$

Indeed, if (4) is true then there is a tight Hamilton cycle $C \cong C_{n}^{(k)}(k-1)$ such that for every pair of its edges $e$ and $f$ with $|e \cap f| \leq \ell$ we have $\phi(e) \neq \phi(f)$. Since, by assumption, $k-\ell$ divides $n, C$ contains a copy of $C_{n}^{(k)}(\ell)$ which is rainbow, as required.

To prove (4) we apply the probabilistic method and Lemma 2.1. To this end, for a given pair $\{e, f\} \in M$ let

$$
Y_{e, f}=\left\{\left\{e^{\prime}, f^{\prime}\right\} \in M:\left\{e^{\prime}, f^{\prime}\right\} \neq\{e, f\} \text { and }(e \cup f) \cap\left(e^{\prime} \cup f^{\prime}\right) \neq \emptyset\right\}
$$

and

$$
X_{e, f}=M \backslash\left(Y_{e, f} \cup\{e, f\}\right) .
$$

To estimate $d$ (cf. (1)), we bound from above the size of $Y_{e, f}$ as follows. For given edges $e$ and $f$ we can find at most $2 k n^{k-1}$ edges $e^{\prime}$ sharing a vertex from $e \cup f$. For every such $e^{\prime}$ we have at most $c n^{k-\ell}$ candidates for $f^{\prime}$, since $e^{\prime}$ and $f^{\prime}$ must have the same color. Thus,

$$
d=\max _{\{e, f\} \in M}\left|Y_{e, f}\right| \leq 2 c k n^{2 k-\ell-1} .
$$

Now, let us consider a uniform probability space consisting of all tight Hamilton cycles $C \cong C_{n}^{(k)}(k-1)$ in $K_{n}^{(k)}$. In order to prove (4), and thus finish the proof of Theorem 1.1, it suffices to show that

$$
\operatorname{Pr}\left(\bigcap_{\{e, f\} \in M} \overline{A_{e, f}}\right)>0 .
$$


Thus, it remains to verify assumption (2) of Lemma 2.1 with $m=|M|, A_{i}:=A_{e, f}$, $X_{i}:=X_{e, f}$, and $Y_{i}:=Y_{e, f}$. Fix $\{e, f\} \in M$ and $X \subseteq X_{e, f}$ and set

$$
\mathcal{C}=\bigcap_{\left\{e^{\prime}, f^{\prime}\right\} \in X} \overline{A_{e^{\prime}, f^{\prime}}}
$$

and

$$
\mathcal{C}_{1}=A_{e, f} \cap \bigcap_{\left\{e^{\prime}, f^{\prime}\right\} \in X} \overline{A_{e^{\prime}, f^{\prime}}} .
$$

In other words, $\mathcal{C}$ is the set of all copies $C$ of $C_{n}^{(k)}(k-1)$ in $K_{n}^{(k)}$ such that $\{g, h\} \not \subset C$ for all $\{g, h\} \in X$, while $\mathcal{C}_{1}=\{C \in \mathcal{C}:\{e, f\} \subset C\}$.

If $\mathcal{C}_{1}=\emptyset$ then the R-H-S of (2) equals zero and there is nothing to prove. Otherwise, we rely on the following technical result the proof of which is postponed to the next section.

Proposition 2.2 For all $1 \leq \ell<k$ there exist constants $\delta=\delta(k, \ell), 0<\delta<1$, such that for every pair e, $f$ of edges of $K_{n}^{(k)}$ with $|e \cap f| \leq \ell$ and for every set $X$ of pairs $g, h$ of edges of $K_{n}^{(k)}$ satisfying $(g \cup h) \cap(e \cup f)=\emptyset$, if $\mathcal{C}_{1} \neq \emptyset$, one can find a disjoint family $\left\{\mathcal{S}_{C}: C \in \mathcal{C}_{1}\right\}$ of sets of copies of $C_{n}^{(k)}(k-1)$ from $\mathcal{C}$ (indexed by the copies $C \in \mathcal{C}_{1}$ ) such that for all $C \in \mathcal{C}_{1}$ we have $\left|\mathcal{S}_{C}\right| \geq \delta n^{2 k-\ell-1}$.

We are now able to specify the constant $c$ by setting

$$
c=\frac{\delta}{10 k},
$$

where $\delta=\delta(k, \ell)$ is the constant given by Proposition 2.2. Then by Proposition 2.2, (5), and (7)

$$
\frac{\operatorname{Pr}\left(A_{e, f} \cap \bigcap_{\left\{e^{\prime}, f^{\prime}\right\} \in X} \overline{A_{e^{\prime}, f^{\prime}}}\right)}{\operatorname{Pr}\left(\bigcap_{\left\{e^{\prime}, f^{\prime}\right\} \in X} \overline{A_{e^{\prime}, f^{\prime}}}\right)}=\frac{\left|\mathcal{C}_{1}\right|}{|\mathcal{C}|} \leq \frac{\left|\mathcal{C}_{1}\right|}{\sum_{C \in \mathcal{C}_{1}}\left|\mathcal{S}_{C}\right|} \leq \frac{1}{\delta n^{2 k-\ell-1}} \leq \frac{1}{4(d+1)} .
$$

Hence, (2) holds and we are in position to apply Lemma 2.1 and conclude that (6) and, consequently, (4) is true. This completes the proof of Theorem 1.1.

Proof of Theorem 1.2. This proof goes along the lines of the proof of Theorem 1.1. Let $c^{\prime}=\frac{\delta}{10 k^{2}}$, where $\delta=\delta(k, \ell)$ is the constant given by Proposition 2.2. Fix a $c^{\prime} n^{k-\ell}$-degree bounded coloring of $K_{n}^{(k)}$. Here we slightly modify the definition of $M$. Let

$$
M=\left\{\{e, f\}: e, f \in K_{n}^{(k)}, \quad 1 \leq|e \cap f| \leq \ell \text { and } \phi(e)=\phi(f)\right\} .
$$

As before,

$$
A_{e, f}=\left\{C \subset K_{n}^{(k)}: C \cong C_{n}^{(k)}(k-1) \text { and }\{e, f\} \subset C\right\} .
$$


and in order to prove Theorem 1.2 it suffices to show that

$$
\bigcap_{\{e, f\} \in M} \overline{A_{e, f}} \neq \emptyset
$$

Indeed, if (8) is true then there is a tight Hamilton cycle $C \cong C_{n}^{(k)}(k-1)$ such that for every pair of its edges $e$ and $f$ with $1 \leq|e \cap f| \leq \ell$ we have $\phi(e) \neq \phi(f)$. Since, by assumption, $k-\ell$ divides $n, C$ contains a copy of $C_{n}^{(k)}(\ell)$ which is properly colored, as required.

We define sets $Y_{e, f}$ and $X_{e, f}$ as before and recalculate the upper bound on $\left|Y_{e, f}\right|$. For given edges $e$ and $f$ we can find at most $2 k n^{k-1}$ edges $e^{\prime}$ sharing a vertex from $e \cup f$. For every such $e^{\prime}$ we have at most $c^{\prime} k n^{k-\ell}$ candidates for $f^{\prime}$ since $e^{\prime}$ and $f^{\prime}$ intersect and have the same color. Thus,

$$
\left|Y_{e, f}\right| \leq 2 c^{\prime} k^{2} n^{2 k-\ell-1} .
$$

The rest of the proof is identical to the proof of Theorem 1.1 and therefore is omitted.

\section{Proof of Proposition 2.2}

Let $e$ and $f$ be given edges in $K_{n}^{(k)}$ such that $|e \cap f| \leq \ell$ and let $C \in \mathcal{C}_{1}$ be a tight Hamilton cycle containing $e$ and $f$ and missing at least one edge from each pair $\{g, h\} \in X$. We describe two constructions depending on the size of $e \cap f$.

Construction 1: for $2 \leq|e \cap f| \leq \ell$.

Let $|e \cap f|=a$ and let $e=\left(u_{1}, \ldots, u_{k}\right)$ and $f=\left(v_{1}, \ldots, v_{k}\right)$ be such that $u_{k-a+1}=$ $v_{1}, u_{k-a+2}=v_{2}, \ldots, u_{k}=v_{a}$. This way we fix an orientation of $C$ where $e$ precedes $f$. Let $P=C \backslash\{e \cup f\}$ be the segment of $C$ between $f$ and $e$ of length $n-2 k+a$. We select arbitrarily $2 k-a-1$ vertex disjoint edges $g_{1}, \ldots, g_{2 k-a-1}$ from $P$, so that $C$ is of the form $e \rightsquigarrow f \rightsquigarrow g_{1} \rightsquigarrow \cdots \rightsquigarrow g_{2 k-a-1} \rightsquigarrow e$, where the symbol $\rightsquigarrow$ indicates a path between the given edges. Clearly, we have $\Omega\left(n^{2 k-a-1}\right)=\Omega\left(n^{2 k-\ell-1}\right)$ choices for the $g_{i}$ 's.

Let $g_{i}=\left(w_{1}^{i}, \ldots, w_{k}^{i}\right)$ for $1 \leq i \leq 2 k-a-1$, where we list the vertices of $g_{i}$ in the order of appearance on $P$. In order to create a cycle $\tilde{C} \in \mathcal{S}_{C}$, we remove all edges which contain at least one vertex from $(e \cup f) \backslash\left\{u_{1}, v_{k}\right\}$ and also all edges whose first vertex (in the order induced by $C$ ) is $w_{j}^{i}$ for $1 \leq i \leq 2 k-a-1$ and $j=1, \ldots, k-1$. After this removal, the vertices in the set $\left\{u_{2}, \ldots, u_{k}, v_{a+1}, \ldots, v_{k-1}\right\}$ become isolated and the remains of the cycle $C$ form a collection of vertex disjoint paths $v_{k} \rightsquigarrow w_{k-1}^{1}, w_{k}^{1} \rightsquigarrow$ $w_{k-1}^{2}, w_{k}^{2} \rightsquigarrow w_{k-1}^{3}, \ldots, w_{k}^{2 k-a-1} \rightsquigarrow u_{1}$.

To create $\tilde{C}$, we connect the above paths by absorbing the isolated vertices. Formally, 


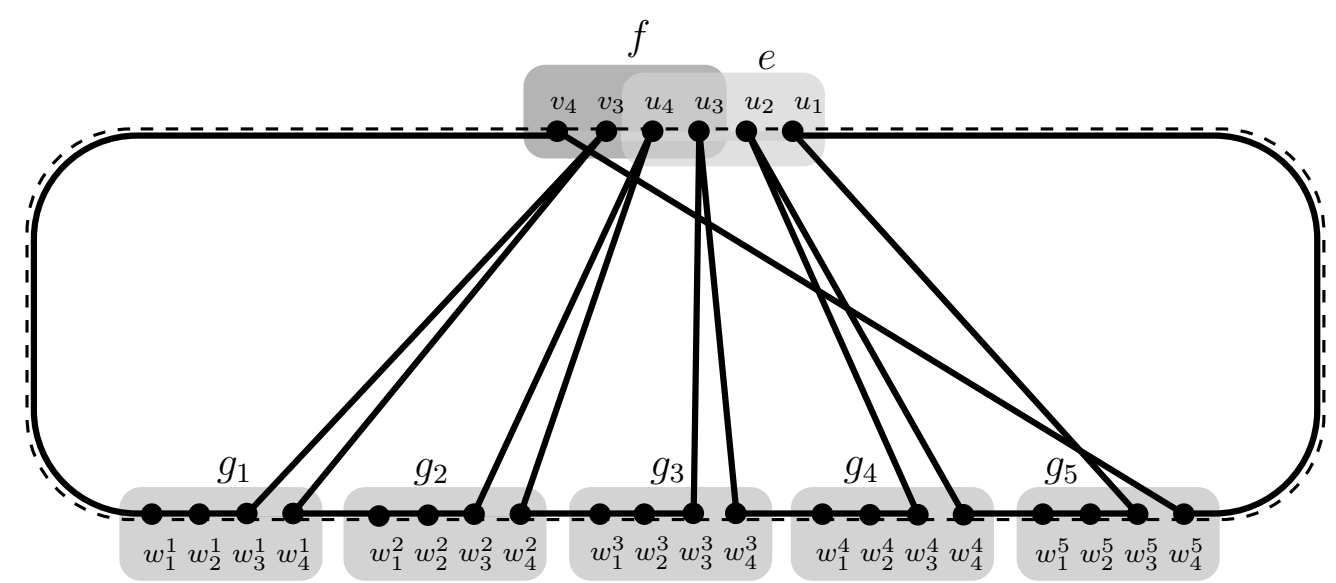

Figure 2: The first construction for $k=4$ and $a=2$. The dashed and solid lines denote $C$ and $\tilde{C}$, respectively.

we define $\tilde{C}$ as the following sequence of vertices (see Fig. 2):

$$
\begin{aligned}
v_{k} & \rightsquigarrow w_{1}^{1}, w_{2}^{1}, \ldots, w_{k-1}^{1}, v_{k-1}, w_{k}^{1} \\
& \rightsquigarrow w_{1}^{2}, w_{2}^{2}, \ldots, w_{k-1}^{2}, v_{k-2}, w_{k}^{2} \\
& \rightsquigarrow \ldots \\
& \rightsquigarrow w_{1}^{k-a-1}, w_{2}^{k-a-1}, \ldots, w_{k-1}^{k-a-1}, v_{a+1}, w_{k}^{k-a-1} \\
& \rightsquigarrow w_{1}^{k-a}, w_{2}^{k-a}, \ldots, w_{k-1}^{k-a}, u_{k}, w_{k}^{k-a} \\
& \rightsquigarrow w_{1}^{k-a+1}, w_{2}^{k-a+1}, \ldots, w_{k-1}^{k-a+1}, u_{k-1}, w_{k}^{k-a+1} \\
& \rightsquigarrow \ldots \\
& \rightsquigarrow w_{1}^{2 k-a-2}, w_{2}^{2 k-a-2}, \ldots, w_{k-1}^{2 k-a-2}, u_{2}, w_{k}^{2 k-a-2} \\
& \rightsquigarrow w_{1}^{2 k-a-1}, w_{2}^{2 k-a-1}, \ldots, w_{k-1}^{2 k-a-1}, u_{1} \rightsquigarrow w_{k}^{2 k-a-1}, v_{k} .
\end{aligned}
$$

It is easy to check that every new edge intersects a vertex from $e \cup f$. Thus, $\tilde{C} \backslash C$ contains no edge from any pair of edges belonging to $X$. Moreover, note that different choices of $g_{i}$ yield different cycles $\tilde{C}$. Thus, $\left|\mathcal{S}_{C}\right|=\Omega\left(n^{2 k-\ell-1}\right)$.

It remains to show that for any two tight Hamilton cycles $C \neq C^{\prime} \in \mathcal{C}_{1}$ we have $\mathcal{S}_{C} \cap \mathcal{S}_{C^{\prime}}=\emptyset$. In order to prove it, one can reverse the above procedure and uniquely determine $C$ and the edges $g_{1}, g_{2}, \ldots, g_{2 k-a-1}$ from $\tilde{C}$. Note that we do not know the order in which the vertices of $e$ and $f$ are traversed by $C$.

To reconstruct $C$, we first find in $\tilde{C}$ a unique $e \rightsquigarrow f$ path $Q$ with no endpoint in $e \cap f$ and exactly on vertex from $e$ and $f$. From this we deduce that $u_{1}=Q \cap e, v_{k}=Q \cap f$ and $w_{k}^{2 k-a-1}$ is the last vertex on $Q$ before $v_{k}$. Now we start at $v_{k}$ and follow $\tilde{C}$ in the direction opposite to $w_{k}^{2 k-a-1}$. Before we reach $u_{1}$ we will intersect $f \cup e$ exactly $2 k-a-2$ times. This way we restore the vertices $v_{k-1}, v_{k-2}, \ldots, v_{a+1}, u_{k}, u_{k-1}, \ldots, u_{2}$ (in the order of appearance on $C$ ). Note that every one of these vertices is adjacent to two vertices $w_{k-1}^{j}$ and $w_{k}^{j}$ for some $1 \leq j \leq 2 k-a-2$. Consequently, we can uncover all edges $g_{i}$ and hence $C$ itself. 
Construction 2: for $|e \cap f| \leq 1$.

Here we show a stronger result, namely, we construct a family $\mathcal{S}_{C}$ of size $\Omega\left(n^{2(k-1)}\right)$. Let $e=\left(u_{1}, \ldots, u_{k}\right)$ and $f=\left(v_{1}, \ldots, v_{k}\right)$. Note that it might happen that $u_{k}=v_{1}$ if $|e \cap f|=1$. Let $P$ be a segment of vertices between $e$ and $f$ of size $\Omega(n)$. For given two vertices $x$ and $y$ denote by $d(x, y)$ the number of vertices on $P$ in the segment between $x$ and $y$. Now we select $2(k-1)$ vertex disjoint edges $g_{1}, \ldots, g_{2(k-1)}$ from $P$ so that $C$ is of the form $e \rightsquigarrow f \rightsquigarrow g_{1} \rightsquigarrow \cdots \rightsquigarrow g_{2(k-1)} \rightsquigarrow e$ and

$$
d\left(v_{k}, w_{k-1}^{1}\right)<d\left(w_{k}^{1}, w_{k-1}^{2}\right)+1<d\left(w_{k}^{2}, w_{k-1}^{3}\right)+1<\cdots<d\left(w_{k}^{k-2}, w_{k-1}^{k-1}\right)+1,
$$

where $g_{i}=\left(w_{1}^{i}, \ldots, w_{k}^{i}\right)$ for each $1 \leq i \leq 2(k-1)$ and the vertices in $g_{i}$ are listed in the order of appearance on $P$. This way we fix an orientation of $C$. (The above sequence of inequalities will be needed later to establish the orientation of $C$ from $\tilde{C}$.) Clearly, we have $\Omega\left(n^{2(k-1)}\right)$ choices for $g_{i}$ 's.

In order to create a cycle $\tilde{C} \in \mathcal{S}_{C}$, we remove all edges which contain at least one vertex from $(e \cup f) \backslash\left\{u_{1}, v_{k}\right\}$ and also all edges whose first vertex (in the order induced by $C$ ) is $w_{j}^{i}$ for $1 \leq i \leq 2(k-1)$ and $j=1, \ldots, k-1$. After this removal, the vertices in the set $\left\{u_{2}, \ldots, u_{k-1}, v_{2}, \ldots, v_{k-1}\right\}$ become isolated and the remains of the cycle $C$ form a collection of vertex disjoint paths $v_{k} \rightsquigarrow w_{k-1}^{1}, w_{k}^{1} \rightsquigarrow w_{k-1}^{2}, w_{k}^{2} \rightsquigarrow w_{k-1}^{3}, \ldots, w_{k}^{2(k-1)} \rightsquigarrow u_{1}$, and $u_{k} \rightsquigarrow v_{1}$. (The latter may be degenerated to the set of isolated vertices.)

To create $\tilde{C}$, we connect the above paths by absorbing the isolated vertices. Formally, we define $\tilde{C}$ as the following sequence of vertices (see Fig. 3):

$$
\begin{aligned}
v_{1}, w_{k-1}^{1}, & w_{k-2}^{1}, \ldots, w_{1}^{1} \rightsquigarrow v_{k}, w_{k}^{1} \\
& \rightsquigarrow w_{1}^{2}, w_{2}^{2}, \ldots, w_{k-1}^{2}, v_{k-1}, w_{k}^{2} \\
& \rightsquigarrow w_{1}^{3}, w_{2}^{3}, \ldots, w_{k-1}^{3}, v_{k-2}, w_{k}^{3} \\
& \rightsquigarrow \ldots \\
& \rightsquigarrow w_{1}^{k-1}, w_{2}^{k-1}, \ldots, w_{k-1}^{k-1}, v_{2}, w_{k}^{k-1} \\
& \rightsquigarrow w_{1}^{k}, w_{2}^{k}, \ldots, w_{k-1}^{k}, u_{k-1}, w_{k}^{k} \\
& \rightsquigarrow w_{1}^{k+1}, w_{2}^{k+1}, \ldots, w_{k-1}^{k+1}, u_{k-2}, w_{k}^{k+1} \\
& \rightsquigarrow \ldots \\
& \rightsquigarrow w_{1}^{2 k-3}, w_{2}^{2 k-3}, \ldots, w_{k-1}^{2 k-3}, u_{2}, w_{k}^{2 k-3} \\
& \rightsquigarrow w_{1}^{2 k-2}, w_{2}^{2 k-2}, \ldots, w_{k-1}^{2 k-2}, u_{1} \rightsquigarrow w_{k}^{2 k-2}, u_{k} \rightsquigarrow v_{1} .
\end{aligned}
$$

It is easy to check that every new edge intersects a vertex from $e \cup f$. Thus, $\tilde{C} \backslash C$ contains no edge from any pair of edges belonging to $X$. Moreover, note that different choices of $g_{i}$ yield different cycles $\tilde{C}$. Thus, $\left|\mathcal{S}_{C}\right|=\Omega\left(n^{2(k-1)}\right)$.

It remains to show that for any two tight Hamilton cycles $C \neq C^{\prime} \in \mathcal{C}_{1}$ we have $\mathcal{S}_{C} \cap \mathcal{S}_{C^{\prime}}=\emptyset$. In order to prove it, one can reverse the above procedure and uniquely determine $C$ and the edges $g_{1}, g_{2}, \ldots, g_{2(k-1)}$ from $\tilde{C}$. Note that we do not know the order in which the vertices of $e$ and $f$ are traversed by $C$. 


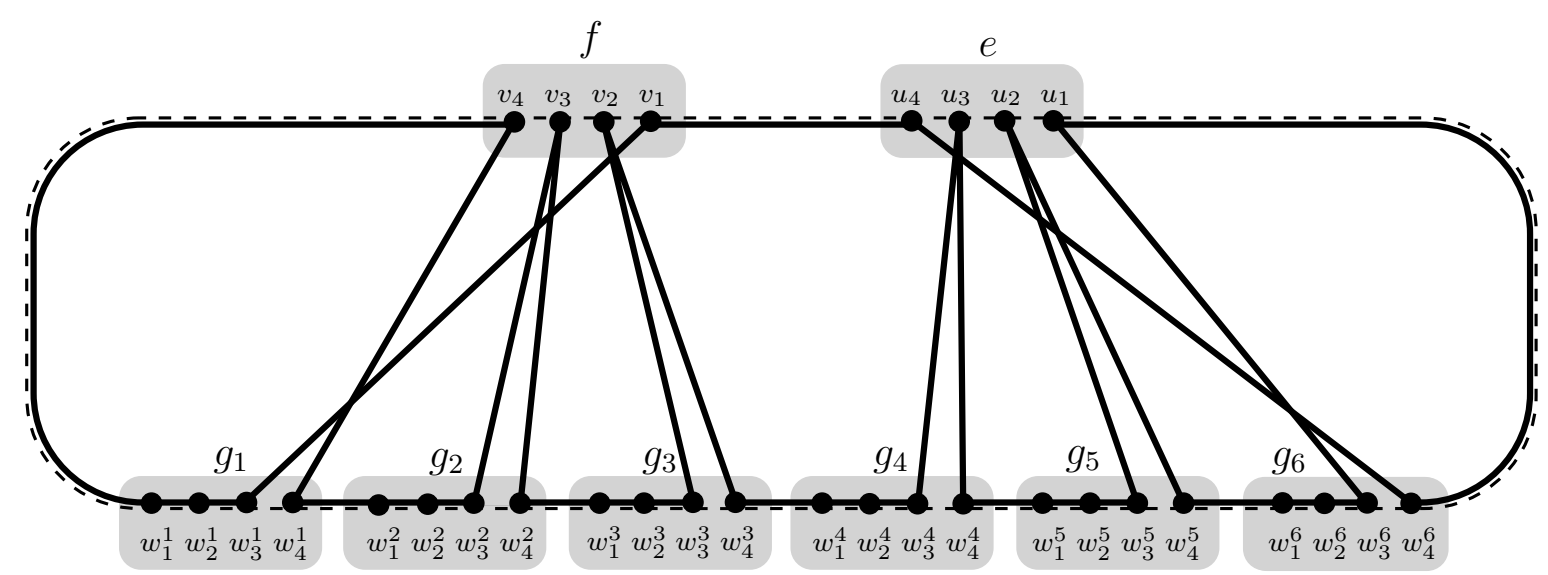

Figure 3: The second construction for $k=4$. The dashed and solid lines denote $C$ and $\tilde{C}$, respectively.

Note that there are exactly two shortest $e \rightsquigarrow f$ paths in $\tilde{C}$, say $Q_{1}$ and $Q_{2}$. One with $u_{k}$ and $v_{1}$ as its endpoints and the second one with $u_{k-1}$ and $v_{2}$ as the endpoints. Our goal is to determine vertex $v_{1}$. Once this is known then as in Construction 1 we can uncover all edges $g_{i}$ and hence $C$ itself.

We assume for a while that $v_{1}=Q_{1} \cap f$. Then we start at $v_{1}$ and follow $\tilde{C}$ in the direction opposite to the second endpoint of $Q_{1}$. Before we reach edge $e$ we will intersect edge $f$ exactly $k-1$ times. This way we pretend that we restore vertices $v_{k}, v_{k-1}, \ldots, v_{2}$ (in the order of appearance). Let $\tilde{d}(x, y)$ be the number of vertices on $\tilde{C}$ between vertices $x$ and $y$. Note that $d\left(v_{k}, w_{k-1}^{1}\right)=\tilde{d}\left(v_{1}, v_{k}\right)-1$ and $d\left(w_{k}^{j}, w_{k-1}^{j+1}\right)=\tilde{d}\left(v_{k-j+1}, v_{k-j}\right)-2$ for $1 \leq j \leq k-2$. Now we check if (9) holds. If so then $Q_{1}$ is really the path with endpoints $u_{k}$ and $v_{1}$; otherwise $Q_{2}$ is the one.

Acknowledgment We would like to thank the referees for their valuable comments and suggestions.

\section{References}

[1] M. Albert, A. Frieze and B. Reed, Multicoloured Hamilton cycles, Electronic Journal of Combinatorics 2 (1995), \#R10.

[2] N. Alon and G. Gutin, Properly colored Hamiltonian cycles in edge-colored complete graphs, Random Structures and Algorithms 11 (1997), 179-186.

[3] J. Bang-Jensen and G. Gutin, Alternating cycles and paths in edge-colored multigraphs: a survey, Discrete Mathematics 165-166 (1997), 39-60.

[4] C. Berge, Nombres de coloration de l'hypergraphe h-parti complet, Springer Lecture Notes in Math. Vol. 411 (1975) 13-20.

[5] B. Bollobás and P. Erdős, Alternating Hamiltonian cycles, Israel Journal of Mathematics 23 (1976), 126-131 
[6] C. Chen and D. Daykin, Graphs with Hamiltonian cycles having adjacent lines different colors, Journal of Combinatorial Theory, Ser. B 21 (1976), 135-139.

[7] A. Dudek and A. Frieze, Tight Hamilton cycles in random uniform hypergraphs, Random Structures \& Algorithms (2012), doi: 10.1002/rsa.20404.

[8] P. Erdős, J. Nešetřil and V. Rödl, Some problems related to partitions of edges of a graph in Graphs and other Combinatorial topics, Teubner, Leipzig (1983), 54-63.

[9] P. Erdős and J. Spencer, Lopsided Lovász local lemma and Latin transversals, Discrete Applied Mathematics 30 (1990), 151-154.

[10] A. Frieze and B. Reed, Polychromatic Hamilton cycles, Discrete Mathematics 118, (1993), 69-74.

[11] A. Gyárfás, G. Sárközy, and E. Szemerédi, Monochromatic Hamiltonian 3-tight Berge cycles in 2-colored 4-uniform hypergraphs, Journal of Graph Theory 63 (2010), 288299.

[12] G. Hahn and C. Thomassen, Path and cycle sub-Ramsey numbers and an edgecolouring conjecture, Discrete Mathematics 62 (1986), 29-33.

[13] A. Johansson, J. Kahn, and V. Vu, Factors in random graphs, Random Structures \& Algorithms 33 (2008), 1-28.

[14] L. Lu and L. Székely, Using Lovász Local Lemma in the space of random injections, Electronic Journal of Combinatorics 14 (2007), \#R63.

[15] V. Rödl and A. Ruciński, Dirac-type questions for hypergraphs - a survey (or more problems for Endre to solve), Bolyai Soc. Math. Studies 21 (2010), An Irregular Mind (Szemerédi is 70), Bolyai Soc. Math. Studies 21 (2010), 561-590.

[16] J. Shearer, A property of the complete colored graph, Discrete Mathematics 25 (1979), $175-178$. 